\title{
Vagus Nerve Stimulation (VNS) vs. Deep Brain Stimulation (DBS) Treatment for Major Depressive Disorder and Bipolar Depression: A Comparative Meta-analytic Review
}

\author{
Ali Mahmood Khan ${ }^{1 *}$, Rizwan Ahmed ${ }^{2}$, Vijaya Padma Kotapati ${ }^{3}$, Sabrina K Dar ${ }^{4}$, Iqra Qamar ${ }^{5}$, Afshan Jafri ${ }^{6}$, \\ Maria Ibrahim ${ }^{7}$, Pawan Kumar ${ }^{8}$, Gulshan Begum ${ }^{9}$
}

\begin{abstract}
Ali Mahmood Khan ${ }^{1 *}$, Rizwan Ahmed², Vijaya Padma Kotapati ${ }^{3}$, Sabrina K Dar Iqra Qamar', Afshan Jafrí, Maria Ibrahim ${ }^{7}$, Pawan Kumar $^{8}$, Gulshan Begum ${ }^{9}$
\end{abstract}

'University of Texas Rio Grande Valley, Texas, USA.

'Liaquat College of Medicine and Dentistry, Karachi, PAKISTAN.

${ }^{3}$ Manhattan Psychiatric Center, New York, USA.

${ }^{4}$ Zucker Hillside Hospital, New York, USA.

${ }^{5}$ Beth Israel Deaconess Medical Center, Massachusetts, USA.

${ }^{6}$ Panjabrao Deshmukh Memorial Medical College, Amravathi, Maharashtra, INDIA.

'Massachusetts Institute of Technology, Boston, USA.

${ }^{8}$ Knapp Medical Center, Weslaco, Texas, USA.

${ }^{9} J a m a i c a$ Hospital Medical Center, 8900 Van Wyck Expy, Richmond Hill, NY 11418, USA

\section{Correspondence}

Dr. Ali Mahmood Khan

University of Texas Rio Grande Valley,

Texas, USA.

Mobile no: +1 408-838-1189

Email: ali_mahmood_khan@hotmail.com

\section{History}

- Submission Date: 08-11-2017

- Revised Date: 30-03-2018

- Accepted Date: 13-09-2018

DOI : 10.5530/ijmedph.2018.3.26

Article Available online

http://www.ijmedph.org/v8/i3

\section{Copyright}

(C) 2018 Phcog.Net. This is an openaccess article distributed under the terms of the Creative Commons Attribution 4.0 International license.

\begin{abstract}
Background: Patients who suffer from major depressive episodes and bipolar disorder often exhibit pharmaco-resistance. Therefore, novel treatment methodologies are being proposed to treat the disease or provide symptomatic relief. VNS and DBS are two such techniques, both of which utilize neurostimulation to achieve therapeutic relief. However, it is necessary to establish the comparative efficacies of these methods in treating MDD in patients. Objective: To assess the relative difference in the efficacy of VNS versus DBS for treatment of Major Depressive Disorder and bipolar depression and to provide evidence for the superior technique. Methods: To compare the efficacy of VNS versus DBS for the reduction of depressive symptoms in patients who meet the criteria for a major depressive episode, we conducted a meta-analysis of studies of the subject. Twenty-six studies were selected, consisting of 1160 patients who were treated with either VNS (Mean age $=47.75$ years old, mean duration of illness $=22.86$ years) or DBS (Mean age $=33.11$ years old, mean duration of illness $=9.9$ years) treatment arms and analyzed them to determine the amount of improvement in mood. The primary outcome measures were evaluated in terms of change between pre-test and post-test scores over a period of three months, as measured by HDRS and MADRS rating scales. Results: A comparison of the summary effect size produced by VNS (HDRS $=1.247$, MADRS $=1.110$ ) to that produced by DBS (HDRS $=2.063$, MADRS $=1.996)$ seems to demonstrate that DBS is the more effective treatment. The effect size for VNS was lower than that of DBS groups, indicating that DBS is more effective than VNS. The finding is corroborated by the tests of heterogeneity; while the VNS group of studies indicated a high level of heterogeneity Vs. DBS group indicated insignificant level of heterogeneity. Conclusion: Current meta-analysis demonstrates that Deep Brain Stimulation (DBS) is a better treatment modality for Major Depressive Disorder and Bipolar Depression than Vagus Nerve Stimulation (VNS). However, as the VNS and DBS groups differed concerning the clinical profiles of the patients (both in terms of age and regarding the duration of the illness. Research studies with larger, synchronous sample sizes and control groups are required for a meta-analysis to draw a steadfast conclusion.
\end{abstract}

Key words: VNS, DBS, Major Depressive Disorder, Bipolar Depression, Neuro-modulation.

\section{INTRODUCTION}

Major depressive disorder (MDD) is a syndrome that deals with both behavior and biology; symptoms include abnormal mood, disturbances in neuro-vegetative functions and a decrease in cognitive abilities and psychomotor functions. It is also referred to as Unipolar Depressive Disorder when it is not accompanied by a history of manic episodes; in cases where there is a history of manic episodes, the condition is referred to as Bipolar Disorder. Manic episodes are characterized by a distinct period of abnormally and persistently elevated, expansive, or irritable mood and abnormally and persistently increased activity or energy, lasting at least four consecutive days and present most of the day, nearly every day. People with the disorder will also sometimes experience mixed episodes in which they experience both high and low moods at the same time or rapidly cycling in the same episode. However, both patients with MDD and those with bipolar disorders are similar in terms of mixed depression states, dysphoric hypomania and family history patterns. Major Depressive Disorder shares some similarities with Bipolar Disorder. Bipolar patients experience includes both mania and depression, whereas persons with major depression (who are not bipolar) will not experience mania. In comparisons of MDD and depressed Bipolar Disorder patients, atypical features, such as hypersomnia or leaden paralysis, psychotic symptoms, psychomotor retardation, shorter depressive episodes, a higher number of depressive recurrences, family history of mood disorders, comorbidity with substance abuse and earlier age at onset are reported more frequently

Cite this article : Khan AM, Ahmed R, Kotapati VP, Dar SK, Qamar I, Jafri A, Ibrahim M, Kumar P, Begum G. Vagus Nerve Stimulation (VNS) vs. Deep Brain Stimulation (DBS) Treatment for Major Depressive Disorder and Bipolar Depression: A Comparative Meta-analytic Review. Int J Med Public Health. 2018;8(3):119-30. 
in Bipolar Disorder, whereas somatic disturbances, anxiety, sleep loss and appetite loss are reported more frequently in MDD. They also evince some similarities: major depressive disorder may shift to bipolar disorder and bipolar disorder shares the feature of depressive states with $\mathrm{Ma}$ jor Depressive Disorder. ${ }^{1-2}$

\section{Treatment approach}

The similarities between MDD and bipolar depression presents some difficulty in determining which treatment modality should be utilized. The first line of treatment for patients suffering from depression includes pharmacotherapies and psychotherapies. The pharmacological treatments include the administration of antidepressant drugs such as selective serotonin reuptake inhibitors and tricyclic anti-depressants, which target specific neurobiological targets. ${ }^{3-4}$ These medications are indicated once the patient begins experiencing depressive states. ${ }^{5}$ Usually the treatment of depression begins with first-line antidepressants such as selective serotonin reuptake inhibitors (fluoxetine, citalopram); if this treatment fails, switching strategies are considered, If that doesn't work; combination strategies are considered (using more than one medication in concert) or augmentation strategies such as psychotherapy, adding other medications (such as bupropion, buspirone, mirtazapine, antipsychotics, T3 thyroid hormone and lithium) and dietary supplements (such as omega-3 fatty acids, S-Adenosyl-L-methionine [SAMe] and folic acid). ${ }^{6-7}$ However, these treatment strategies may not always reap desired results, such as in the case of treatment-resistant disorders, resistance which often shows itself with major depressive disorders and the depressive state of bipolar disorder. These disorders are characterized by the failure of antidepressant and augmentation therapies to bring relief to the patient, resulting in demand for better treatment methods for the management of the condition. ${ }^{8}$

The advanced field of neurobiology has associated the occurrence of depressive states, such as MDD and bipolar depression, with abnormalities in the functioning of neural networks. The neural network theory states that a normal mood is regulated by means of a coordinated neural structural that is functional normally. The occurrence of depressive states has been speculated to arise from communication of a dysfunctional nature between the nodes of different brain regions. ${ }^{9-10}$ Neuroimaging studies have indicated certain consistent and overlapping brain abnormalities in MDD and bipolar patients, such as an enlargement of the limbic regions, ventricle volume and increased and hyper-intensive rates of sub-cortical gray matter functioning. Thus, the presentation of biological models concerning depression has led to the advent of novel treatment methodologies that target the neural systems concerning the functional clusters of cognition, motivation, homeostasis and emotional regulation. ${ }^{11}$

The term neuromodulation techniques (NTs) refers to one such group of methods that involve targeting specific neural structures to facilitate improvement in functionality and they have proven effective in treating treatment-resistant disorders. ${ }^{9}$ These techniques involve invasive, minimally invasive, or non-invasive techniques that stimulate cortical or sub-cortical regions of the brain for therapeutic purposes. ${ }^{12}$ There are a number of NTs adopted for treating MDD such as Transcranial magnetic stimulation (rTMS), Vagus nerve stimulation (VNS), Deep brain stimulation (DBS), Transcranial direct current stimulation (rTDS), Direct cortical stimulation (DCS) and Magnetic seizure therapy. ${ }^{13-14}$ VNS and DBS constitute the invasive category, whereas rTMS, tDCS, DCS and MST constitute the group of techniques in the non-invasive category.

VNS involves the electrical stimulation of the tenth cranial nerve with a miniature implantable neurostimulator such as a Bionic Neuron that can be implanted (with a minor surgical procedure) adjacent to one or more portions of the vagus nerve and that manipulate the pathological substrate so as to achieve the desirable therapeutic effect. ${ }^{15}$ The VNS generator contains a small battery in the device that generates an intermittent electrical stimulation to the vagus nerve. A surgeon implants the generator subcutaneously over the chest and attaches the electrodes to the left vagus nerve. Intermittent signals from the VNS device travel up the vagus nerve and enter the medulla. ${ }^{16}$

DBS, on the other hand, involves the electrical stimulation of particular regions of the brain like the subgenual anterior cingulate cortex via electrodes, most commonly using four-contact stimulating electrodes. ${ }^{17}$ However, both techniques include the stimulation of specific neurological centers to provide relief from symptoms. The non-invasive techniques involve the application of electromagnetic waves to relevant brain regions to modulate activity and provide relief. ${ }^{18}$

\section{Need for study}

Treatment-resistant depression could lead to deleterious consequences for patients because not treating the disorder can contribute to the global burden of disease, impairing the structural and functional capacity of the brain and rendering the individual incapable of social functioning. ${ }^{8}$ There are a number of treatment modalities available for providing relief to patients suffering from treatment-resistant MDD and bipolar depression. Proposed theories regarding the neurobiological basis of disease progression has led to the incorporation of several neuromodulation techniques such as transcranial magnetic stimulation, Vagus nerve stimulation, deep brain stimulation, magnetic seizure therapy and others in the treatment of these conditions. The present study is motivated by increasing evidence of the efficacy and safety of VNS and the similar treatment methodology of DBS in improving depression. ${ }^{14,19}$ The present study is undertaken to provide a comparative view of the two stimulation techniques by evaluating the relative difference in the efficacy of these two methodologies. Thus, the aim of the present study is to perform a meta-analysis of treatment results pertaining to VNS and DBS. The objective of the study is to identify the superior method among two (VNS vs. DBS) for treating patients suffering from MDD and bipolar depression based on analytic review of the relevant research.

\section{METHODOLOGY}

\section{Search strategy}

For the purpose of extracting relevant literature for the present metaanalysis, different electronic databases were searched. The investigated databases included PubMed, EMBASE, Cochrane Central Register of Controlled Trials and PubMed Clinical Queries; the time frame referenced for this study is last 20 years (until the time of the search in June 2017). Additionally, a manual search of references from previously published meta-analyses focused upon either DBS or VNS was also performed. The set of keywords used are listed in Table 1.

\section{Inclusion Criteria}

The criteria listed below were designated to collect relevant studies fulfilling the aim of the present research:

- $\quad$ Studies published in the English language.

- Studies involving only treatment-resistant depression, either/both Unipolar (MDD) and Bipolar Treatment-Resistant Depression.

- The studies contained clear descriptions of clinical outcomes utilizing validated outcome measures.

- Studies reporting treatment with respect to Studies reporting pre- and post-treatment scores.

\section{Exclusion Criteria}

The exclusion criteria were designated to minimize the inclusion of non-pertinent information sources. The following list details exclusion criteria: 


\begin{tabular}{|c|c|}
\hline Individual Keywords & Combined Keywords \\
\hline VNS & VNS TRD \\
\hline TRD & DBS TRD \\
\hline $\begin{array}{c}\text { DBS } \\
\text { Vagus Nerve Stimulation }\end{array}$ & $\begin{array}{c}\text { Vagus Nerve Stimulation Treatment-Resistant } \\
\text { Depression }\end{array}$ \\
\hline $\begin{array}{l}\text { Vagus Nerve Stimulation } \\
\text { Treatment-Resistant } \\
\text { Depression }\end{array}$ & $\begin{array}{c}\text { Deep Brain Stimulation Treatment-Resistant } \\
\text { Depression }\end{array}$ \\
\hline Deep Brain Stimulation & \\
\hline
\end{tabular}

- Studies which included brain stimulation techniques other than VNS and DBS.

- $\quad$ Studies where DBS or VNS used as adjunctive therapies.

- Studies reporting patients with other mental illnesses such as bipolar illness (manic or rapid cyclic), schizoaffective disorders, or others.

- $\quad$ Case reports, gray literature

\section{Selection of studies}

Initially, searches were conducted to find studies using VNS and DMS in patients with MDD/Unipolar depression. The initial search produced only two and seven studies for VNS and DBS, respectively. As the search can only be built to be inclusive and not exclusive, the search results included participants with bipolar depression. To better understand the effectiveness of VNS and DMS in affective disorders, the decision was made to incorporate studies that included patients with bipolar depression. The final search resulted in a total of 25 studies for the systematic review. Bipolar depression and MDD are studied together in published literature in the form of meta-analyses to better understand the effectiveness of treatment modalities..$^{20}$ Out of the 25 studies, nine were selected for conducting a meta-analysis, of which five examined VNS and four examined DBS. The relevant studies were selected on the basis of homogeneity of the treatment outcome (pre-post scores) presented by the studies, over the three-month acute period, to facilitate extraction of valid statistical results.

\section{Data extraction}

The papers selected included significant information regarding the two different treatment methods; hence, uniformity was maintained in extracting the data from the different studies. The following structure was followed to extract the information:

1. Patient characteristics: Age, gender, duration of illness, criteria for diagnosis and definition of treatment-resistant depression.

2. Measure of primary outcome: The Hamilton Depression Rating Scale (HDRS) and Montgomery-Åsberg Depression Rating Scale (MADRS) scores, both pre- and post-neuro-modulation treatment were recorded and provided an operational measure of mood improvement. Besides HDRS and MADRS scores, the number of participants who were identified as improving, based on an efficacy measure of $\geq 50 \%$ reduction in HDRS score post-treatment, were noted.

3. Measures of secondary outcome: The pre- and post-neuro-modulation treatment scores for the secondary measures were also recorded, at different time points, by the researchers (CGI-I, GAF, BDI, BAI, IDS-SR, YMRS).

4. The stimulation parameters and adverse events due to treatment procedure were also extracted.

\section{Data synthesis and analysis}

The systematic review was conducted in agreement with the PRISMA guidelines, ${ }^{21}$ accompanied by a statistical meta-analysis procedure performed using the Comprehensive meta-analysis software (CMA v3, Englewood, NJ, USA). The meta-analysis was conducted using random effects modeling, as it accounts for the assumption of difference in effect sizes between the different studies and the summary effect size is the estimate of the mean of a random sample of effect sizes. The standard difference in means was calculated using the continuous data of treatment scores reported on HDRS and MADRS scales. For the studies reporting two groups, the data from the group receiving active VNS or DBS treatment was used.

The heterogeneity among the different studies was reported using the I-square test statistic, with heterogeneity among the studies evaluated as a measure of the statistic value. ${ }^{22}$ The studies were also analyzed for publication bias by using funnel plots as well as Begg and Mazumdar's and Egger's linear regression test statistics. The outlying effect sizes, if any, were identified using sensitivity analysis, which involved removing the studies having greatest effect size values.

\section{Literature search and screening}

The studies included in the final meta-analysis were searched and screened following the methodology depicted in Figure 1.

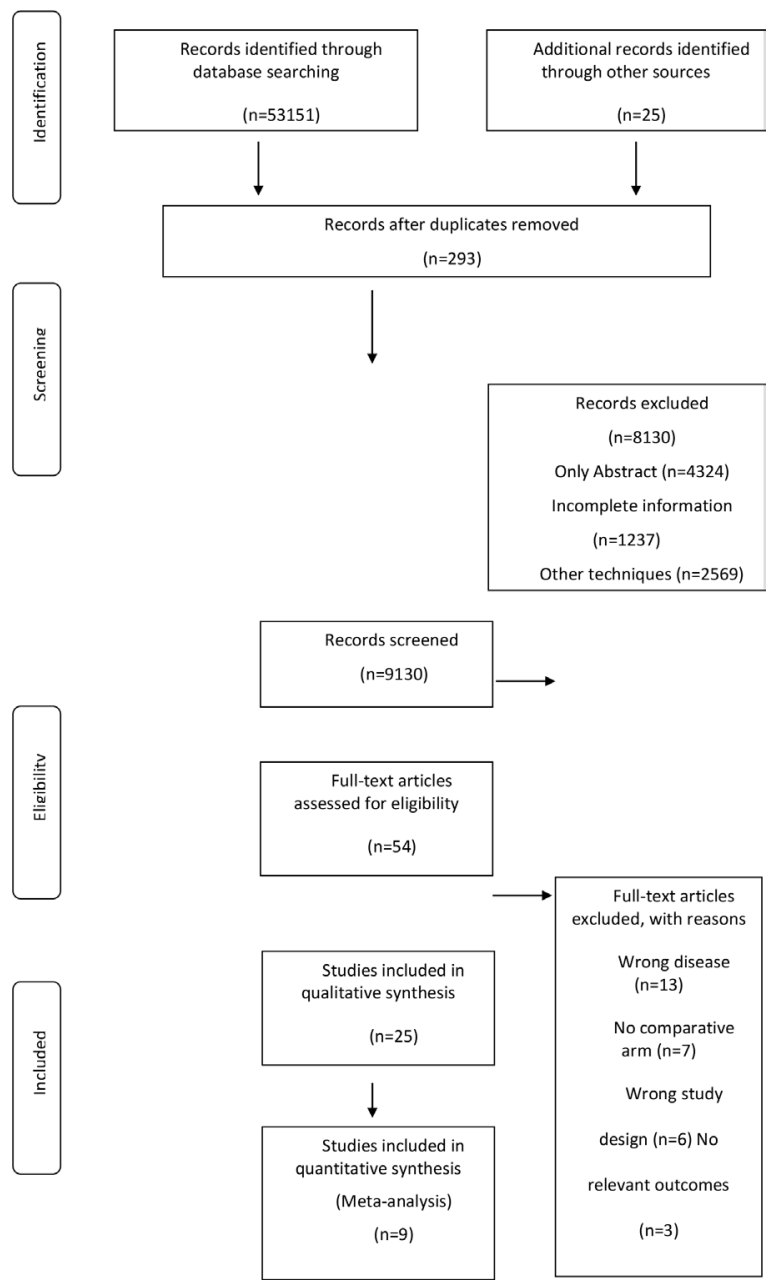

Figure 1: Systematic review flowchart. 


\section{Systematic review}

The systematic review involved the collection of studies for Vagus nerve stimulation and Deep brain stimulation techniques of neuro-modulation administered to the patients demonstrating treatment-resistant depression. Table 1 describes the characteristics of the studies included in the present systematic review; the VNS review included twelve studies for the period from year 2000 to 2017 and the DBS review included thirteen studies for the period between the years of 2005 and 2017. The studies pertaining to both the NTs presented different study designs. For VNS, six open-label trials and a single study each of long-term follow-up study, comparative study, randomized control trial, prospective study, double-blind study and observational study design were included in the systematic review. For DBS, seven open-label trials, one randomized control trial and one prospective study each were included. Additionally, there were four DBS studies using combined research designs.

\section{Characteristics of studies}

A. VNS : As shown in Table 2, the VNS studies were largely conducted in the US and Germany. ${ }^{29-30}$ The studies included the early postoperative follow-up and the follow-up period. The early post-operative follow-up included all of the patients participating in the study and they were monitored by investigators. The follow-up period varied from study to study. During this follow-up period, improvement in condition was monitored, but not all participants could be followed. The VNS studies included patients suffering from bipolar and unipolar depressive disorders; care was taken to ensure to include only bipolar patients not currently in a rapid cycling stage and not currently suffering a major depressive episode.

The Vagus nerve stimulation technique was clinically tested on patients suffering from both MDD and bipolar disorder and has proven to be a powerful treatment option. ${ }^{47}$

B. DBS: Studies of DBS have been conducted with fair geographical distribution, spread across the US , Canada, ${ }^{35-36,43}$ Germany, Spain and the Netherlands. ${ }^{46}$ The DBS studies were also characterized by different acute study and follow-up periods. After device implantation, the individual studies continued for varying periods of time. Also, the majority of DBS trials took into account only those patients suffering from major depressive or unipolar disorder, except for four studies which included the only bipolar patients in the total sample.

\section{Patient Characteristics}

Table 3 shows the characteristics of patients included in the VNS and DBS studies. The VNS studies included 1743 patients, which included 1213 females and 530 males. Their mean age was 47.75 years and the average duration of illness was 22.86 years. The DBS studies included a total of 165 patients, with 98 females and 67 males, a mean age of 33.11 years and an average duration of illness of 9.9 years. The DBS sample size was very small compared to the sample size of the VNS trials, which could possibly be attributed to the relatively recent emergence of this technique for treating depression..$^{48}$

\section{Treatment Outcomes}

Table 4 shows the primary treatment outcomes for both the VNS and DBS trials. The Hamilton Depression Rating Scale (HDRS) and Montgomery-Asberg Depression Rating Scale (MADRS) were designated as the primary outcome measures, whereas IDS-C, YMRS, CGI, GAF all reported secondary outcome measures. Both HDRS and MADRS are the most commonly used rating scales for evaluating depression, using established clinical criteria to differentiate between severity levels of the condition and to measure the evolution and recovery from the depressive episode. ${ }^{49}$ The two scales have also been found to be correlated to each other to a significant degree, suggesting the two scales measure depression similarly, with only slight differences. ${ }^{50-51}$ Although some studies criticize the HDRS scale as being biased and argue that the MADRS is superior in evaluating the condition, HDRS has continued to be the gold standard rating scale for the past 40 years. ${ }^{30}$

a) VNS: The VNS trials reported improvement in the depressive states of patients as seen in the reduction in post-test scores. The average values of simulation parameters bringing about relief in the condition of patients could not be calculated, as some of the studies did not report the stimulation parameters. Reported side effects were observed in all of the studies, side effects associated with the implantation and operation of the VNS device. Voice alteration or hoarseness was reported to be the most common side effect of VNS, both of which could be caused by compromised airways in the larynx. ${ }^{52}$ Other reported side effects associated with device implantation were pain at the site of incision, infection, throat pain, neck and general pain, shortness of breath, headache, dyspnea, pharyngitis, dysphagia, asystole, bradycardia and discomfort. The VNS stimulation led to a significant degree of improvement, yet some studies reported suicidal ideation as well as suicides and suicide attempts in some patients. The research conducted by Aaronson et al. ${ }^{32}$ studied the effects of VNS at three different settings: low (Current $=0.25 \mathrm{~mA}$, Pulse width $=130$ $\mu \mathrm{sec}$ ), medium (Current $=0.5-1.0 \mathrm{~mA}$, Pulse width $=250 \mu \mathrm{sec}$ ) and high (Current $=1.25-1.5 \mathrm{~mA}$, Pulse width $=250 \mu \mathrm{sec}$ ). Nine to eleven percent of patients in the medium and high groups showed remission compared to a five to six percent remission rate in the group using low settings.

b) DBS: The DBS trials, despite small sample sizes, demonstrated significant improvement in the depressive states of patients. The average amplitude of the administered treatment was calculated to be $4.03 \mathrm{~V}$, the average pulse width was $127.69 \mathrm{~ms}$ and the average frequency was $118.26 \mathrm{~Hz}$. Besides the different stimulation parameters, the DBS studies focused on stimulating different areas of the brain, areas that are thought to play an important role in the development and maintenance of depression. The different brain regions stimulated by researchers in the studies to date are the subgenual cingulate white matter, the ventral capsule/ventral striatum, ${ }^{14,53}$ the nucleus accumbens, ${ }^{38-39}$ the subgenual cingulate gyrus and the superolateral branch of the forebrain bundle. ${ }^{45}$ However, determining the most effective brain region will require more research and trials. DBS was found to have a higher number of side effects, with unique effects such as psychomotor slowing at high settings, hand numbness, aconuresis and cephalalgia. There were other general side effects including infection. The most severe reported side effect was suicidal ideation.

The study conducted by Riva-Posse et al. ${ }^{44}$ aimed at validating the subcallosal cingulate region as an effective site to stimulate in reducing depressive states by using a four-bundle tractography 'connectome blueprint' to plan surgical targeting in participants. Ramasubbu et al..$^{43}$ discusses identifying the optimal procedure for stimulating parameters and evaluating optimized stimulation parameters by using a research design consisting of a double-blind stimulus optimization phase and an open-label post-optimization phase. Puigdemont et al. ${ }^{40}$ successfully demonstrated full remission in four patients out of eight in total after a complete year of stimulation. They also noted that the localization of electrodes is an important parameter in eliciting a response, with electrodes localized in the BA24 region, the corpus callosum and the head of caudate producing the highest response. All of the studies agreed that the positioning of electrodes and the stimulation parameters play a crucial role in treating depression. 


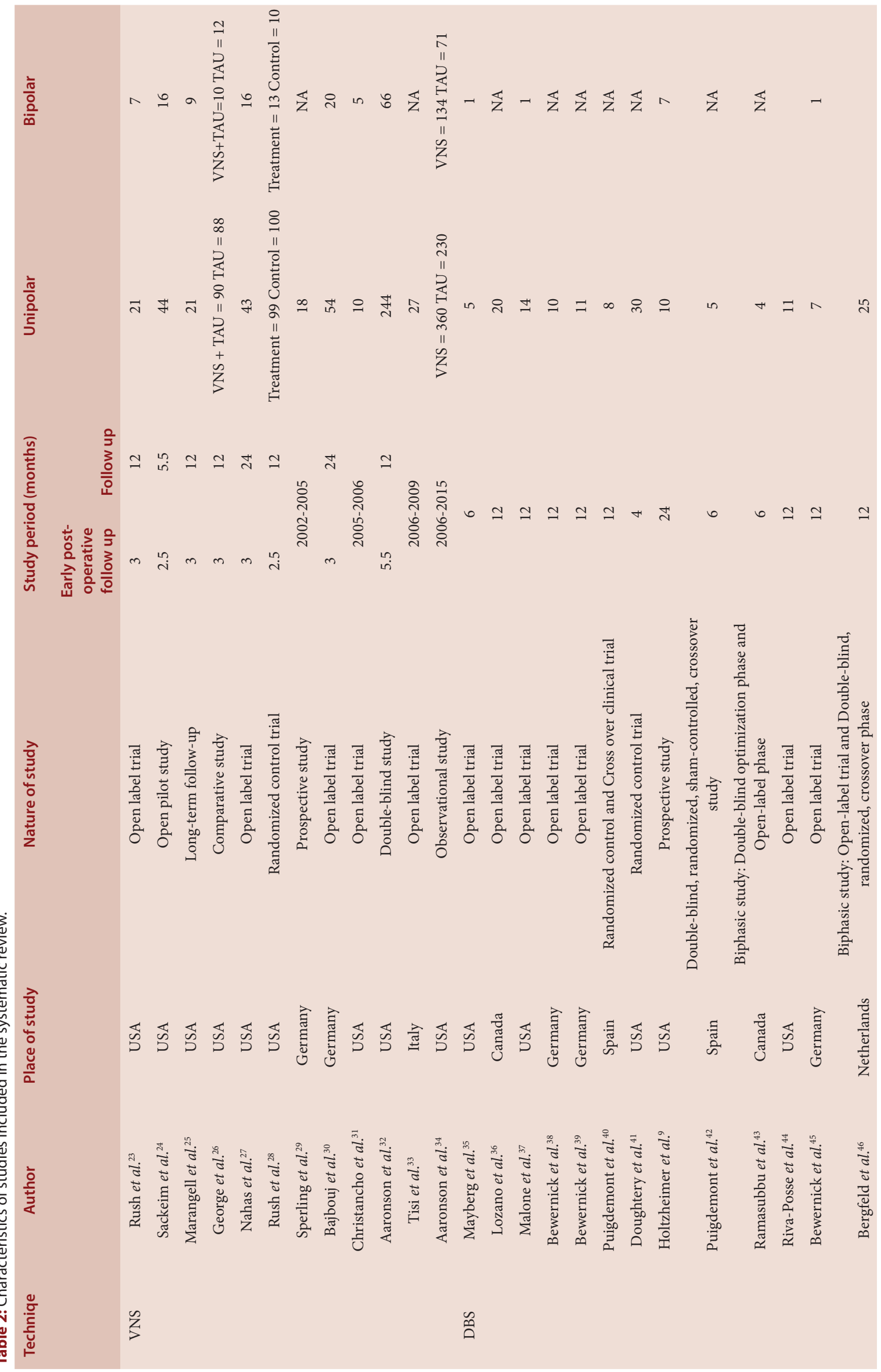




\begin{tabular}{|c|c|c|c|c|c|}
\hline Technique & Author & Age & Gender (F/M) & $\begin{array}{l}\text { Duration of illness/current } \\
\text { episode }\end{array}$ & $\begin{array}{l}\text { Duration of } \\
\text { intervention }\end{array}$ \\
\hline \multirow[t]{12}{*}{ VNS } & Rush et al..$^{23}$ & $47.5 \pm 7.5$ & $20 / 10$ & $19.3 \pm 13.1$ & 3 months \\
\hline & Sackeim et al. ${ }^{24}$ & $46.8 \pm 8.7$ & $39 / 21$ & $18.1 \pm 10.9$ & 3 months \\
\hline & Marangell et al..$^{25}$ & NA & NA & NA & 12 months \\
\hline & George et al..$^{26}$ & $\begin{array}{c}\mathrm{VNS}+\mathrm{TAU}=21.8 \pm 11.9 \\
\mathrm{TAU}=20.8 \pm 11.5\end{array}$ & $\begin{array}{c}\mathrm{VNS}+\mathrm{TAU}=64 / 36 \\
\mathrm{TAU}=69 / 32\end{array}$ & $\begin{array}{c}\mathrm{VNS}+\mathrm{TAU}=25.5 \pm 11.9 \mathrm{TAU} \\
=25.8 \pm 13.2\end{array}$ & NA \\
\hline & Nahas et al..$^{27}$ & $46.8 \pm 8.7$ & $38 / 21$ & $46.8 \pm 8.7$ & 24 months \\
\hline & Rush et al..$^{28}$ & $\begin{array}{c}\text { Treatment }=47 \pm 9 \text { Control }= \\
45.9 \pm 9\end{array}$ & $\begin{array}{c}\text { Treatment }=66 / 46 \\
\text { Control }=73 / 37\end{array}$ & $\begin{aligned} \text { Treatment } & =26.10 \pm 11 \text { Control } \\
& =24.9 \pm 13.0\end{aligned}$ & 3 months \\
\hline & Sperling et al. ${ }^{29}$ & $\begin{aligned} \text { Treatment } & =50.2 \pm 8.5 \text { Control } \\
& =50 \pm 8.8\end{aligned}$ & 5F/4M (both) & $\begin{aligned} \text { Treatment } & =7.2 \pm 1.9 \text { Control } \\
& =6.9 \pm 0.8\end{aligned}$ & NA \\
\hline & Bajbouj et al..$^{30}$ & $47.4 \pm 11.7$ & $50 / 24$ & $19.1 \pm 10.5$ & 24 months \\
\hline & Christancho et al. ${ }^{31}$ & $49 \pm 10$ & $9 \mathrm{~F} / 6 \mathrm{M}$ & $31.7 \pm 11.1$ & NA \\
\hline & Aaronson et al..$^{32}$ & $\begin{array}{c}\text { Low dosage }=49.1 \pm 10.5 \\
\text { Medium dosage }=47.2 \pm 11 \\
\text { High dosage }=47.4 \pm 10.8\end{array}$ & $\begin{array}{c}\text { Low dosage }=68 / 34 \\
\text { Medium dosage }= \\
69 / 32 \text { High dosage } \\
=73 / 34\end{array}$ & $\begin{array}{c}\text { Low dosage }=29.8 \pm 12.1 \\
\text { Medium dosage }=26.3 \pm 10.9 \\
\text { High dosage }=27.0 \pm 12.1\end{array}$ & NA \\
\hline & Tisi et al..$^{33}$ & $57.5 \pm 14$ & $9 \mathrm{~F} / 18 \mathrm{M}$ & $18.5 \pm 13.3$ & 24 months \\
\hline & Aaronson et al..$^{34}$ & $\begin{aligned} \mathrm{VNS}= & 48.9 \pm 10.12 \mathrm{TAU}= \\
& 49.9 \pm 11.07\end{aligned}$ & $\begin{aligned} \mathrm{VNS} & =350 / 144 \mathrm{TAU} \\
& =211 / 90\end{aligned}$ & NA & NA \\
\hline \multirow[t]{14}{*}{ DBS } & Mayberg et al..$^{35}$ & $29.5 \pm 12$ & $3 \mathrm{~F} / 3 \mathrm{M}$ & $29.5 \pm 12$ & 6 months \\
\hline & Lozano et al. ${ }^{36}$ & $27.1 \pm 8.3$ & $11 \mathrm{~F} / 9 \mathrm{M}$ & $27.1 \pm 8.3$ & 12 months \\
\hline & Malone et al..$^{37}$ & $25.3 \pm 10.5$ & $11 \mathrm{~F} / 4 \mathrm{M}$ & $25.3 \pm 10.5$ & 12 months \\
\hline & Bewernick et al..$^{38}$ & $48.6 \pm 11.7$ & $4 \mathrm{~F} / 6 \mathrm{M}$ & $48.6 \pm 11.7$ & 24 months \\
\hline & Bewernick et al..$^{39}$ & $48.36 \pm 11.08$ & $4 \mathrm{~F} / 7 \mathrm{M}$ & $48.36 \pm 11.08$ & 24 months \\
\hline & Puigdemont et al..$^{40}$ & $24.9 \pm 5.3$ & $6 \mathrm{~F} / 2 \mathrm{M}$ & $24.9 \pm 5.3$ & 6 months \\
\hline & & & & Active $=46.6$ & NA \\
\hline & Doughtery et al. ${ }^{41}$ & Active $=46.6$ Control $=48.9$ & $17 / 13$ & Control $=48.9$ & \\
\hline & Holtzheimer et al. ${ }^{9}$ & $42 \pm 8.9$ & $10 \mathrm{~F} / 7 \mathrm{M}$ & $42 \pm 8.9$ & NA \\
\hline & Puigdemont et al. ${ }^{42}$ & $\begin{aligned} \text { OFF-ON } & =27.5 \pm 0.7 \text { ON-OFF } \\
& =20.3 \pm 2.5\end{aligned}$ & & $\begin{aligned} \text { OFF-ON } & =27.5 \pm 0.7 \mathrm{ON}-\mathrm{OFF} \\
& =20.3 \pm 2.5\end{aligned}$ & 6 months \\
\hline & Ramasubbu et al. ${ }^{43}$ & $17.25 \pm 5.0$ & $3 \mathrm{~F} / 1 \mathrm{M}$ & $17.25 \pm 5.0$ & 6 months \\
\hline & Riva-Posse et al..$^{44}$ & $48.73 \pm 10.10$ & $9 \mathrm{~F} / 2 \mathrm{M}$ & $48.73 \pm 10.10$ & 12 months \\
\hline & Bewernick et al..$^{45}$ & $41.9 \pm 8.70$ & $3 \mathrm{~F} / 5 \mathrm{M}$ & $41.9 \pm 8.70$ & NA \\
\hline & Bergfeld et al. ${ }^{46}$ & $53.2 \pm 8.4$ & $17 / 8$ & $53.2 \pm 8.4$ & NA \\
\hline
\end{tabular}

\section{Meta-analysis}

Effect Size

The present meta-analytic review evaluates the treatment efficacy of two neuromodulation techniques, VNS and DBS, as established by published research. The treatment designs recorded improvement in terms of pre- and post-test scores, with treatment administered to a single group of patients. To observe the reported efficacy of individual treatment arms, the meta-analysis was conducted separately for each technique and the summary effect sizes were compared to determine the efficacy and sensitivity of each treatment. ${ }^{54} \mathrm{~A}$ higher value of summary effect size indicates a higher degree of improvement in scores, thus the associated efficacy of the treatment methodology.
HDRS: The summary effect size (standard difference in means) for VNS and DBS groups was 1.247 and 2.063 respectively, as shown in Figure 2. These figures indicate that DBS is more effective than VNS, as reported by the analyzed studies, during the early postoperative follow-up (three months) in treating depression. With respect to heterogeneity, the VNS group of studies had the value of $\mathrm{I}^{2}$ statistic of $87.46, \mathrm{p}=0.00$, indicating $(84.76 \%)$ a high level of significant heterogeneity among the studies, whereas the DBS group of studies had the value of $\mathrm{I}^{2}$ statistic of 0.00 , but $\mathrm{p}=0.740$, indicating insignificant heterogeneity among the studies. ${ }^{22}$ MADRS: The summary effect size (standard difference in means) for VNS and DBS groups was 1.110 and 1.996 respectively, as shown in Figure 3. This indicated the higher efficacy of DBS over VNS, as reported 


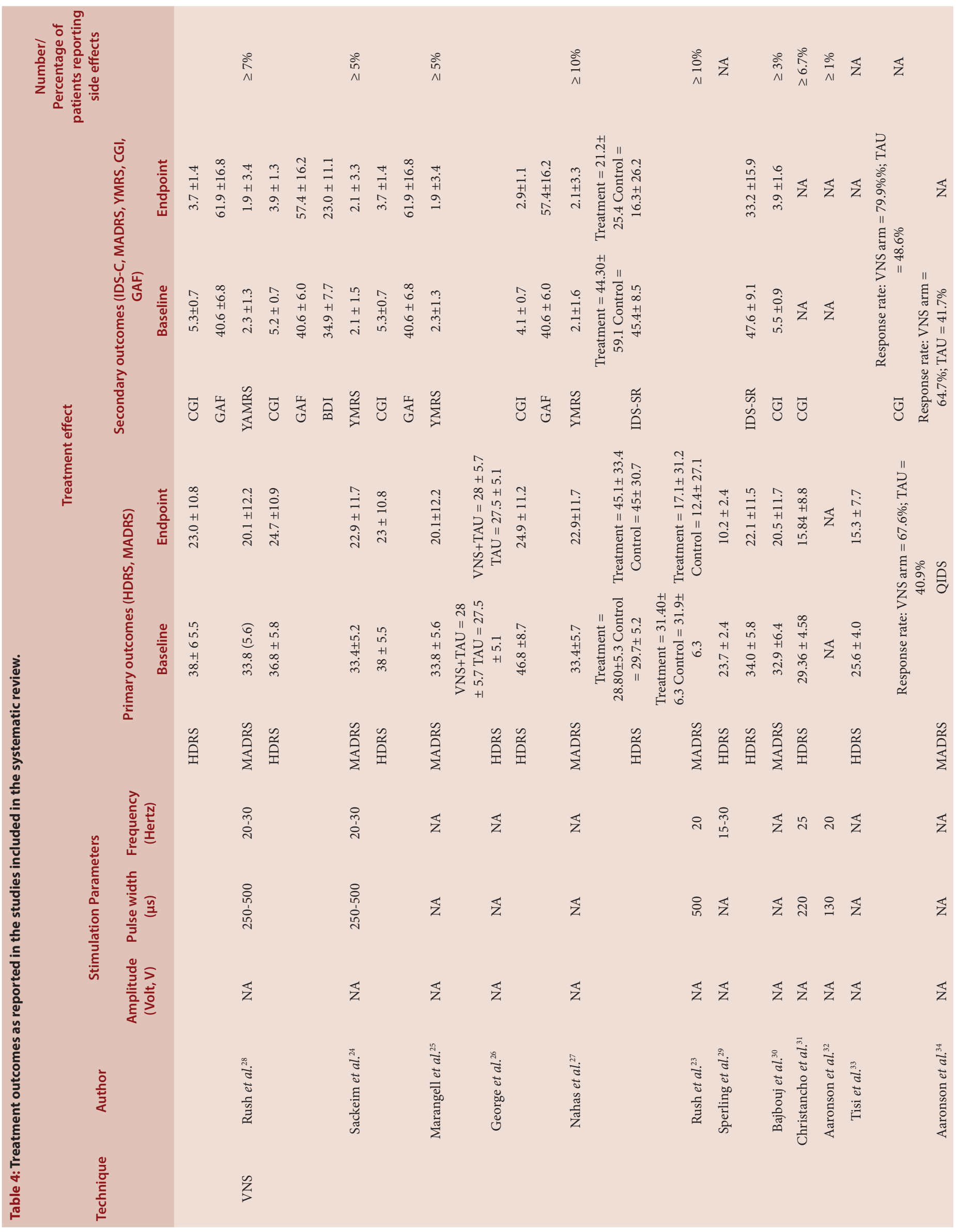




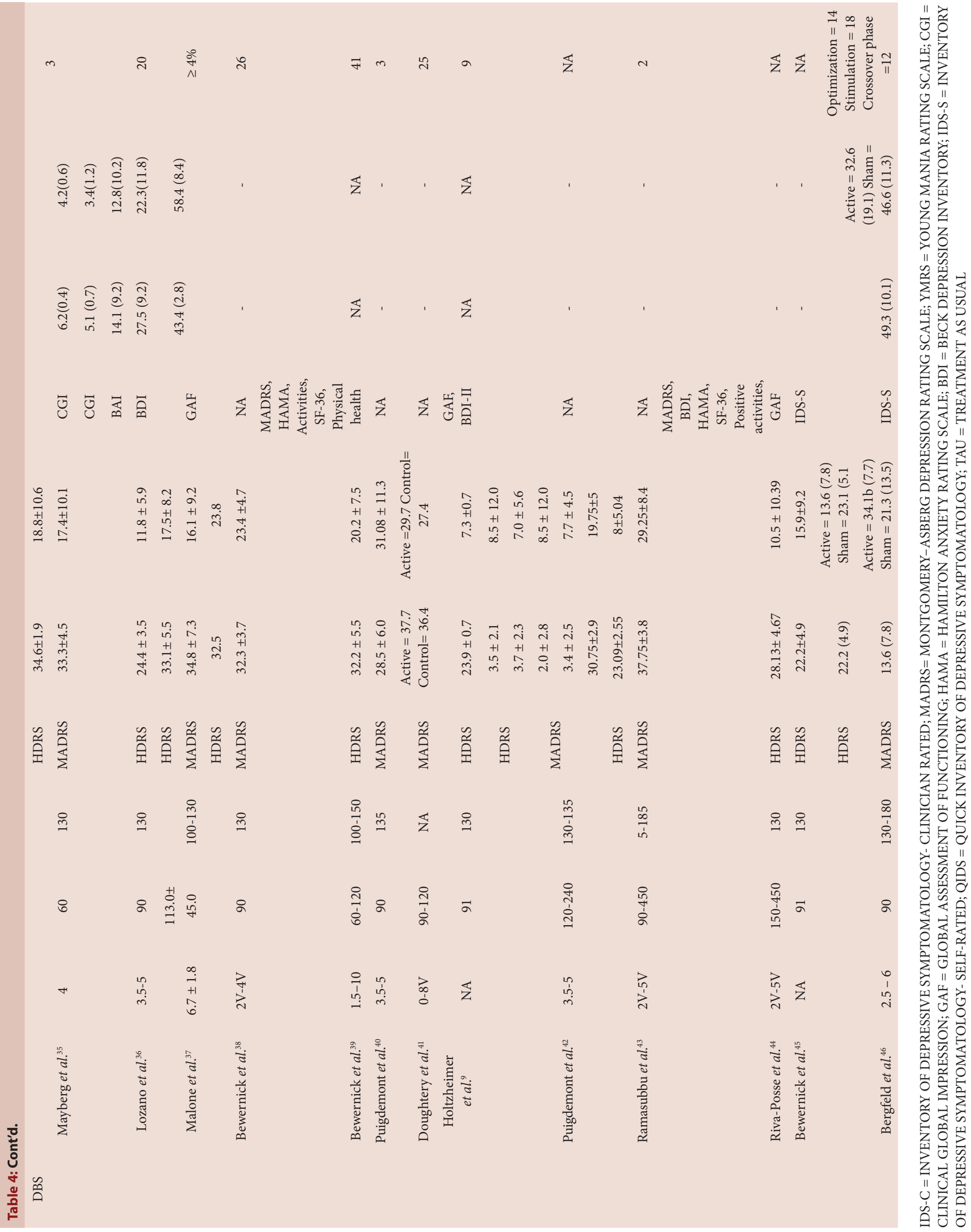



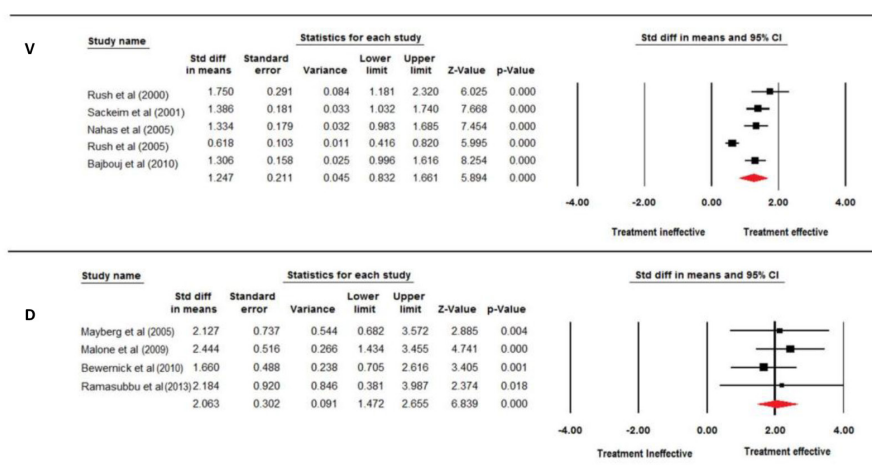

Figure 2: Forest plot for VNS and DBS treatment scores with respect to the HDRS rating scale.
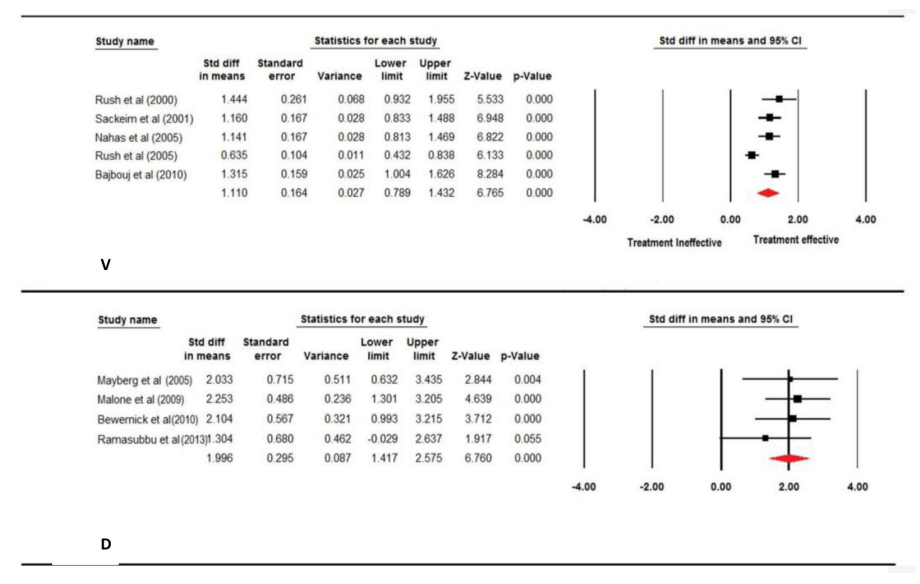

Figure 3: Forest plot for VNS and DBS treatment scores with respect to MADRS rating scale.

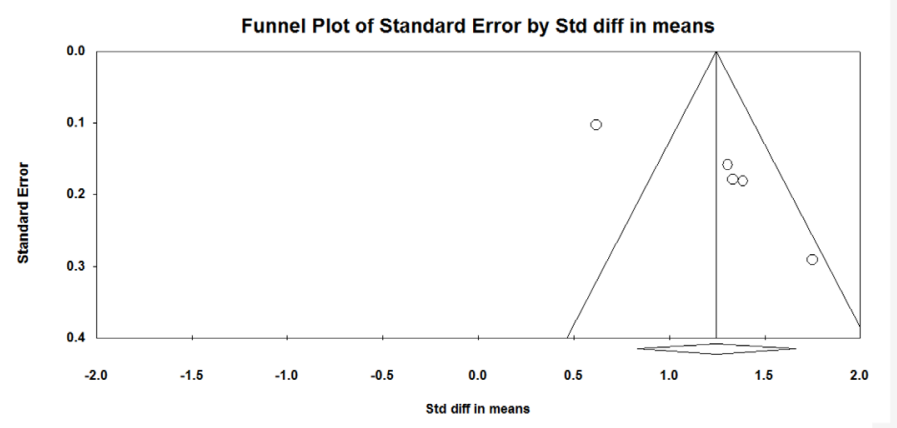

Figure 4: Funnel plot for VNS studies.

by studies, during the early post-operative follow-up (three months), in treating depression. With respect to heterogeneity, the VNS group of studies had the value of $\mathrm{I}^{2}$ statistic of $80.75, \mathrm{p}=0.00$, indicating $(80.75 \%)$ a high level of significant heterogeneity among the studies, whereas the DBS group of studies had the value of $\mathrm{I}^{2}$ statistic of 0.00 , but $\mathrm{p}=0.761$, indicating an insignificant heterogeneity among the studies. ${ }^{22}$

\section{Publication Bias}

The data suffered from inherent bias pertaining to study designs, as the age and duration of the illness of the subjects in VNS and DBS groups were not comparable. Also, the VNS publications were older than the DBS publications.

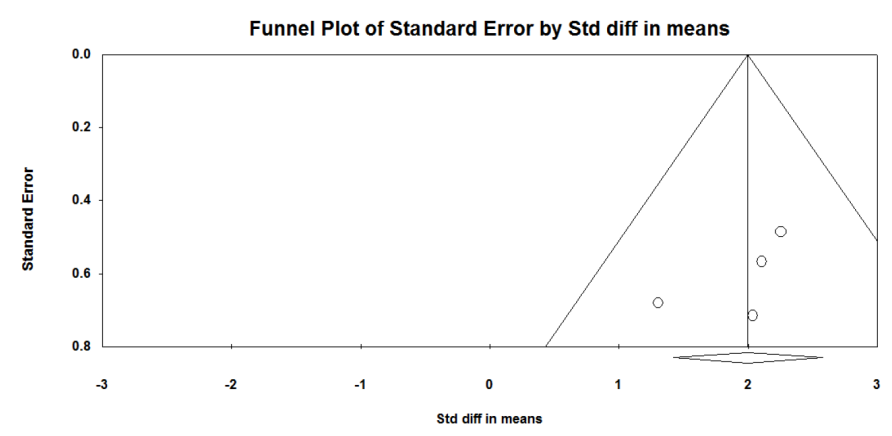

Figure 5: Funnel plot for DBS studies.

a) VNS: The publication bias was tested and the funnel plot was obtained, depicted in Figure 4. The plot was asymmetrical, with Egger's rank test showing an intercept $=6.43, \mathrm{p}=0.04$, indicating the presence of bias.

b) DBS: We tested the publication bias was tested obtained the funnel plot, which is depicted in Figure 5. The plot was asymmetrical, with Egger's rank test showing an intercept of $-2.59, \mathrm{p}=0.31$, indicating symmetry in funnel plot and an insignificant publication bias.

\section{DISCUSSION}

Vagus Nerve Stimulation (VNS) and Deep Brain Stimulation (DBS) both involve electrical stimulation of the neural target via an implanted device..$^{55}$ The constant electrical stimulation results in the alteration of the activity of the targeted brain area; depending upon the location of the implant, these NTs have proven to be effective in bringing relief to patients suffering from MDD and bipolar depression. ${ }^{56}$

VNS involves the placement of a pulse-generating device subcutaneously wrapped around the left Vagus nerve, with the stimulatory effect resulting in changes in neurotransmitters such as serotonin, glutamate, norepinephrine and GABA, all of which are indicated in depression pathogenesis and improving mood. This treatment also brings about changes in the anatomy of brain regions and blunts mild stress events that may eventually result in stress sensitization in the individual. ${ }^{57}$

DBS involves invasive surgery and the electrode placement varies. This variation has been a topic of research: different studies have targeted different areas of brain, such as the Subcingulate-Broadmann area 25, the Ventral anterior internal capsule/ventral striatum (VC/VS), the Nucleus accumbens (NAcc), the Inferior thalamic peduncle and the Lateral habenula. ${ }^{14}$ Though DBS has been known to improve mood in depression, the technique is still being tested to determine which brain areas obtain optimal results.

The stimulation of Nucleus accumbens (NAcc) has been observed to play a significant role in the abnormal reward process, whereas VC/VS stimulation has been shown to improve concomitant symptoms of depression in patients treated with OCD. Even though VNS and DBS have proved themselves to significantly improve the symptoms of MDD and bipolar depression, these methods are also associated with certain shortcomings. The foremost drawback associated with both is the expense of treatment, the special aftercare required, the invasive nature of procedure and the risk of hemorrhages. ${ }^{58}$ These techniques are also associated with certain diverse events such as infections due to implants, neck pain, dyspnea, dysphagia, vomiting, voice alteration, headache and other conditions. ${ }^{59}$

The two techniques vary significantly in their mode of action: VNS has been demonstrated to act upon the prefrontal cortex and limbic structures, whereas the mode of action of DBS is still under investigation. The 
deeply invasive nature of this technique has been associated with its action on areas far beyond the targeted region. Thus, it has been associated with putative modeling of the complex neural networks and is perceived to influence larger volumes of neural tissue, depending upon the stimulation parameters. ${ }^{60}$ The mode of action of the two techniques is yet to be understood completely, even using advanced neuroimaging techniques.

The clinical studies are still in their nascent stage and researchers continue to establish the safety and efficacy of these treatment methods. The results of the individual VNS and DBS meta-analyses has shown that these treatment methods indeed provide benefit, even in patients who previously exhibited high refractory responses with previous drug and ECT treatments. The long periods of follow-up in VNS treatment provide evidence for the stability of the technique in bringing long-term relief to patients, as the study outcomes showed continued improvement in scores even after a period of 12-24 months. Although DBS has shown promising results, the results suffer limitation in terms of sample size and randomized controlled data. ${ }^{61}$

The invasive nature of the treatment methodologies causes some patients to be reluctant to undergo such treatments; however, in spite of recorded side effects, patients generally tolerate the surgery and continued stimulation well. A major effort is being directed towards the optimization of the stimulation parameters, a consideration that is even more important using DBS because different areas of the brain are structured differently and have relatively different impedance profiles. The stimulation of different brain areas involves impacting different surface areas and thus different charge densities are required. Overall, VNS and DBS are suitable treatment methods for providing relief to patients with mental illnesses.

The present meta-analytic review has compared the relative efficacies of the two emerging neurostimulation treatment methodologies for depression, namely VNS and DBS. The present study is the first one to attempt such a comparison of these techniques. The comparison of the summary effect sizes showed the superiority of DBS over VNS in ameliorating depression.

The summary effect size for VNS was lower than that of DBS groups, indicating that DBS method of brain stimulation is more effective than VNS. The finding is corroborated by the tests of heterogeneity: while the VNS group of studies indicated a high level of significant heterogeneity among the studies, the DBS group indicated insignificant level of heterogeneity. Thus, it may be that DBS is more efficacious than VNS. Additionally, the presence of publication bias in the case of VNS and the insignificant publication bias in the DBS studies further supports the claim that DBS is more efficient than VNS. Thus, the current meta-analysis demonstrates that Deep Brain Stimulation (DBS) is a better treatment modality for Major Depressive Disorder and Bipolar Depression than Vagus Nerve Stimulation (VNS).

However, as the clinical profile of patients in the VNS group and DBS group were very different in terms of age for treatment of MDD and bipolar depression, the duration of the illness and the duration of follow-up or intervention, it is relatively difficult to make direct comparison between these two treatment interventions. To be able to make these comparisons, it is necessary to have reasonably matched studies (both in terms of participants and in terms of methodologies). Since such matched studies do not exist in published literature, it may not be practical to interpret the difference in effect size reported from the meta-analysis as being evidence for one being a better treatment.

\section{Limitations}

The present study suffers from certain inherent limitations in terms of the studies included and in terms of statistical procedures. The foremost limitation is that the results generated from the present study could not be extrapolated to a general, large-scale population, as studies that were analyzed included research with very small sample sizes, especially the DBS studies. The clinical profile of patients in the VNS group and DBS group were very different in terms of age, duration of illness and duration of follow up or intervention. Another limitation is that these techniques are relatively new in the domain of psychiatric procedures and the complete implications are not yet known. Additionally, the VNS trials included bipolar patients (though they were also in a depressive state) with those suffering from major depression; therefore, it is unclear how effective VNS is for treating unipolar depressive states. (The present review includes only two studies ${ }^{29,33}$ in which the VNS technique was used with only one clinical group.) Therefore, the comparison was, in some ways, comparing apples to oranges: studies of unipolar patients were compared with studies that included both unipolar and bipolar patients. And while the results of the statistical analysis and subsequent comparison of summary effect size have indicated that DBS is the superior treatment methodology, the disparity between the sample sizes of VNS and DBS also posed a limitation to the conclusions drawn from the meta-analysis. This led to the biggest limitation: because the studies included different sample sizes (and the samples themselves were so different), because the types and durations of the treatments varied and because one group was more homogenous than the other, direct comparisons could not be made. Thus, while the summary effects were different, it is not clear that these are very conclusive.

\section{Implications of research}

The treatment of mental illnesses is a fertile area of research and the increase in refractory responses in patients with depression calls for improved treatment methods. This meta-analysis seems to indicate that DBS is a more effective treatment. However, the present review is a pilot review subject to limitations. Those limitations can and should be utilized as a framework for outlining problems in the research and suggesting the types of research that should be done, calling future researchers to delve deeper into the problem and present concrete evidence. The analysis has suggested that DBS can improve major depressive states through the stimulation of brain regions. However, an investigation into the region of the brain producing the highest level of response is required, with simultaneous optimization of stimulation parameters. Also, there is a need for controlled trials to confirm the efficacy of treatment in diagnosed patients as compared to control groups. The stimulation parameters may also function as criteria for customized therapy for non-responders, allowing the adjustment of the parameters in order to obtain suitable responses. The invasive nature of these stimulation techniques requires close monitoring of patient, however, as these techniques have not been tested on a larger scale with large numbers of people and the associated cognitive and physiological effects have yet to be identified.

\section{CONCLUSION}

The VNS and DBS techniques have been found to be suitable for treating major depression. The long-term follow-up periods of some of these studies have indicated that these methods may provide long-term, sustained and stable relief for patients. The stimulation parameters and positioning of electrodes were also seen as predictors of response; in other words, some techniques and positioning proved more effective than others. However, studies with larger sample sizes and synchronous experimental and control groups are required. There is a need for studies whose participants are more similar in age with similar durations of the illness and a need for studies which match in terms of the type of stimulation used and the duration of the treatment. However, these methods are showing promising results and taken together with suitable 
medications, they could be used in the management of treatment-resistant depression.

\section{CONFLICT OF INTEREST}

The authors declare no conflict of interest.

\section{ABBREVIATIONS}

VNS: Vagus Nerve Stimulations; DBS: Deep Brain Stimulation; MDD: Major Depressive Disorder; TRD: Treatment Resistant Depression; ECT: Electro Convulsive Therapy; OCD: Obsessive Compulsive Therapy; TAU: Treatment As Usuals; GA: Generalized Anxiety; rTMS: Transcrania Magnetic Stimulation; NTs: Neuromodulation Techniques; DCS: Direct Cortical Stimulation; rTDS: Transcranial Direct Current stimulation; GABA: Gamma-Aminobutyric Acid; tDCS: Transcranial Direct-current Stimulation; MST: Magnetic secure Transmission; BAI: Beck Anxiety Inventory; IDS-C: Inventory of Depressive Symptomatology- Clinician Rated; MADRS: Montgomery Asberg Depression Dating Scale; YMRS: Young Mania Rating Scale; CGI-I: Clinical Global Impression- Improvement; GAF: Global Assessment of Functioning; HAMA: Hamilton Anxiety Rating Scale; BDI: Beck Depression Inventory; IDSSR: Inventory of depressive symptomatology- self-rated; QIDS: Quick Inventory of Depressive Symptomatology.

\section{SUMMARY}

In this study we assessed the relative difference in the efficacy of VNS versus DBS for treatment of MDD and bipolar depression and to provide evidence for the superior technique. 26 studies were selected, consisting of 1160 patients who were treated with either VNS or DBS treatment arms and analyzed them to determine the amount of improvement in mood and primary outcome measures were evaluated in terms of change between pre-test and post-test scores over a period of three months, as measured by HDRS and MADRS rating scales. Results comparing effect size produced by VNS (HDRS $=1.247$, MADRS $=1.110)$ to that produced by DBS (HDRS $=2.063$, MADRS $=1.996$ ) seems to demonstrate that DBS is the more effective treatment, while the VNS group of studies indicated a high level of heterogeneity Vs. DBS group indicated insignificant level of heterogeneity. However, as the VNS and DBS groups differed concerning the clinical profiles of the patients both in terms of age and regarding the duration of the illness. Research studies with larger, synchronous sample sizes and control groups are required for a meta-analysis to draw a steadfast conclusion.

\section{REFERENCES}

1. Benazzi F. The relationship of major depressive disorder to bipolar disorder: continuous or discontinuous?. Current Psychosis and Therapeutics Reports. 2005;3(3):131-9

2. Tsuang MT, Faraone SV. The genetics of mood disorders: Johns Hopkins University Press. 1990.

3. Association AP. Practice guideline for the treatment of patients with bipolar disorder (revision): American Psychiatric Pub. 2002.

4. Kupfer DJ, Frank E, Phillips ML. Major depressive disorder: new clinical, neurobiological and treatment perspectives. The Lancet. 2012;379(9820):1045-55.

5. Willner P, Scheel KJ, Belzung C. The neurobiology of depression and antidepressant action. Neuroscience and Biobehavioral Reviews. 2013;37(10):2331-71.

6. Moret $\mathrm{C}$. Combination/augmentation strategies for improving the treatment of depression. Neuropsychiatric Disease and Treatment. 2005;1(4):301.

7. Schwartz T, Rashid A. Augmentation and Combination Pharmacotherapy trends in major depressive disorder: Results of a brief survey of psychiatrists. P AND T. 2007;32(1):28.

8. Al-Harbi KS, Qureshi NA. Neuromodulation therapies and treatment-resistant depression. Medical Devices (Auckland, NZ). 2012;5:53.

9. Holtzheimer PE, Mayberg HS. Neuromodulation for treatment-resistant depression. F1000 Medicine Reports. 2012;4.

10. Wessa M, Kanske P, Linke J. Bipolar disorder: a neural network perspective on a disorder of emotion and motivation. Restorative Neurology and Neuroscience.
2014;32(1):51-62.

11. Linden DE. Neurofeedback and networks of depression. Dialogues in Clinical Neuroscience. 2014;16(1):103.

12. Rossi S, Santarnecchi E, Valenza G, Ulivelli M. The heart side of brain neuromodulation. Phil Trans R Soc A. 2016;374(2067):20150187.

13. Brunoni AR, Teng CT, Correa C, Imamura M, Brasil NJP, Boechat R, et al. Neuromodulation approaches for the treatment of major depression: challenges and recommendations from a working group meeting. Arquivos De Neuro-Psiquiatria. 2010;68(3):433-51.

14. Cusin C, Dougherty DD. Somatic therapies for treatment-resistant depression: ECT, TMS, VNS, DBS. Biology of Mood and Anxiety Disorders. 2012;2(1):14.

15. Boon P, Vonck K, Herdt VD, Van DA, Goethals M, Goossens L, et al. Deep brain stimulation in patients with refractory temporal lobe epilepsy. Epilepsia. 2007;48(8):1551-60

16. George MS, Aston JG. Non-invasive techniques for probing neurocircuitry and treating illness: vagus nerve stimulation (VNS), transcranial magnetic stimulation (TMS) and transcranial direct current stimulation (tDCS). Neuropsychopharmacology. 2010;35(1):301.

17. Herrington TM, Cheng JJ, Eskandar EN. Mechanisms of deep brain stimulation. Journal of Neurophysiology. 2015;115(1):19-38.

18. Bain L, Norris SP, Stroud C. Non-Invasive Neuromodulation of The Central Nervous System. 2015.

19. Perlmutter JS, Mink JW. Deep brain stimulation. Annu Rev Neurosci. 2006;29:229-57.

20. Lee EE, Della SMP, Liu A, Himelhoch S. Ketamine as a novel treatment for major depressive disorder and bipolar depression: a systematic review and quantitative meta-analysis. General Hospital Psychiatry. 2015;37(2):178-84.

21. Liberati A, Altman DG, Tetzlaff J, Mulrow C, Gøtzsche PC, loannidis JP, et al. The PRISMA statement for reporting systematic reviews and meta-analyses of studies that evaluate health care interventions: explanation and elaboration. PLoS Medicine. 2009;6(7):e1000100.

22. Higgins JP, Green S. Cochrane handbook for systematic reviews of interventions: John Wiley and Sons. 2011.

23. Rush AJ, George MS, Sackeim HA, Marangell LB, Husain MM, Giller C, et al. Vagus nerve stimulation (VNS) for treatment-resistant depressions: a multicenter study. Biological Psychiatry. 2000;47(4):276-86.

24. Sackeim HA, Rush AJ, George MS, Marangell LB, Husain MM, Nahas Z, et al. Vagus nerve stimulation (VNS ${ }^{\mathrm{TM}}$ ) for treatment-resistant depression: efficacy, side effects and predictors of outcome. Neuropsychopharmacology. $2001 ; 25(5): 713-28$.

25. Marangell LB, Rush AJ, George MS, Sackeim HA, Johnson CR, Husain MM, et al. Vagus nerve stimulation (VNS) for major depressive episodes: one year outcomes. Biological Psychiatry. 2002;51(4):280-7.

26. George MS, Rush AJ, Marangell LB, Sackeim HA, Brannan SK, Davis SM, et al. A one-year comparison of vagus nerve stimulation with treatment as usual for treatment-resistant depression. Biological Psychiatry. 2005;58(5):364-73.

27. Nahas Z, Teneback C, Chae JH, Mu Q, Molnar C, Kozel FA, et al. Serial vagus nerve stimulation functional MRI in treatment-resistant depression. Neuropsychopharmacology. 2007;32(8):1649.

28. Rush AJ, Marangell LB, Sackeim HA, George MS, Brannan SK, Davis SM, et al. Vagus nerve stimulation for treatment-resistant depression: a randomized, controlled acute phase trial. Biological Psychiatry. 2005;58(5):347-54.

29. Sperling W, Reulbach $U$, Kornhuber J. Clinical benefits and cost effectiveness of vagus nerve stimulation in a long-term treatment of patients with major depression. Pharmacopsychiatry. 2009;42(03):85-8.

30. Bajbouj M, Merkl A, Schlaepfer TE, Frick C, Zobel A, Maier W, et al. Two-year outcome of vagus nerve stimulation in treatment-resistant depression. Journal of Clinical Psychopharmacology. 2010;30(3):273-81.

31. Cristancho P, Cristancho MA, Baltuch GH, Thase ME, O'Reardon JP. Effectiveness and safety of vagus nerve stimulation for severe treatment-resistant major depression in clinical practice after FDA approval: outcomes at 1 year. The Journal of Clinical Psychiatry. 2011

32. Aaronson ST, Carpenter LL, Conway CR, Reimherr FW, Lisanby SH, Schwartz TL, et al. Vagus nerve stimulation therapy randomized to different amounts of electrical charge for treatment-resistant depression: acute and chronic effects. Brain Stimulation: Basic, Translational and Clinical Research in Neuromodulation. 2013;6(4):631-40.

33. Tisi G, Franzini A, Messina G, Savino M, Gambini O. Vagus nerve stimulation therapy in treatment-resistant depression: A series report. Psychiatry and Clinical Neurosciences. 2014;68(8):606-11.

34. Aaronson ST, Sears P, Ruvuna F, Bunker M, Conway CR, Dougherty DD, et al. A 5-year observational study of patients with treatment-resistant depression treated with vagus nerve stimulation or treatment as usual: comparison of response, remission and suicidality. American Journal of Psychiatry. 2017; 174(7):640-8.

35. Mayberg HS, Lozano AM, Voon V, McNeely HE, Seminowicz D, Hamani C, et al. Deep brain stimulation for treatment-resistant depression. Neuron. 2005;45(5):651-60. 
36. Lozano AM, Mayberg HS, Giacobbe P, Hamani C, Craddock RC, Kennedy SH. Subcallosal cingulate gyrus deep brain stimulation for treatment-resistant depression. Biological Psychiatry. 2008;64(6):461-7.

37. Malone Jr DA, Dougherty DD, Rezai AR, Carpenter LL, Friehs GM, Eskandar EN, et al. Deep brain stimulation of the ventral capsule/ventral striatum for treatment-resistant depression. Biological Psychiatry. 2009;65(4):267-75.

38. Bewernick BH, Hurlemann R, Matusch A, Kayser S, Grubert C, Hadrysiewicz B, et al. Nucleus accumbens deep brain stimulation decreases ratings of depression and anxiety in treatment-resistant depression. Biological Psychiatry. 2010;67(2):110-6.

39. Bewernick BH, Kayser S, Sturm V, Schlaepfer TE. Long-term effects of nucleus accumbens deep brain stimulation in treatment-resistant depression: evidence for sustained efficacy. Neuropsychopharmacology. 2012;37(9):1975.

40. Puigdemont D, Pérez ER, Portella MJ, Molet J, Diego ADJ, Gironell A, et al. Deep brain stimulation of the subcallosal cingulate gyrus: further evidence in treatment-resistant major depression. International Journal of Neuropsychopharmacology. 2012;15(1):121-33.

41. Dougherty DD, Rezai AR, Carpenter LL, Howland RH, Bhati MT, O’Reardon JP et al. A randomized sham-controlled trial of deep brain stimulation of the ventral capsule/ventral striatum for chronic treatment-resistant depression. Biological Psychiatry. 2015;78(4):240-8.

42. Puigdemont D, Portella MJ, Pérez ER, Molet J, Gironell A, Diego ADJ, et al. A randomized double-blind crossover trial of deep brain stimulation of the subcallosal cingulate gyrus in patients with treatment-resistant depression: a pilot study of relapse prevention. Journal of Psychiatry and Neuroscience: JPN. 2015;40(4):224.

43. Ramasubbu R, Anderson S, Haffenden A, Chavda S, Kiss ZH. Double-blind optimization of subcallosal cingulate deep brain stimulation for treatmentresistant depression: a pilot study. Journal of Psychiatry and Neuroscience: JPN. 2013;38(5):325.

44. Riva PP, Choi K, Holtzheimer PE, Crowell AL, Garlow SJ, Rajendra JK, et al. A connectomic approach for subcallosal cingulate deep brain stimulation surgery: prospective targeting in treatment-resistant depression. Molecular Psychiatry. 2018;23(4):843.

45. Bewernick BH, Kayser S, Gippert SM, Switala C, Coenen VA, Schlaepfer TE Deep brain stimulation to the medial forebrain bundle for depression-long-term outcomes and a novel data analysis strategy. Brain Stimulation. 2017;10(3):664-71.

46. Bergfeld IO, Mantione M, Hoogendoorn ML, Ruhé HG, Notten P, Laarhoven JV, et al. Deep brain stimulation of the ventral anterior limb of the internal capsule for treatment-resistant depression: a randomized clinical trial. JAMA Psychiatry. 2016;73(5):456-64

47. Gelziniene G, Seeck M. Vagus Nerve Stimulation in Depression. Richtlinien für die Autoren.177.

48. Sommers RP, Dings R, Neijenhuijs KI, Andringa H, Arts S, Bult DVD, et al. A young scientists' perspective on DBS: a plea for an international DBS organization. Neuroethics. 2015;8(2):187-90.

49. Snaith R. Present use of the Hamilton Depression Rating Scale: observation on method of assessment in research of depressive disorders. The British Journal of Psychiatry. 1996;168(5):594-7.

50. Cusin C, Yang H, Yeung A, Fava M. Rating scales for depression. Handbook of Clinical Rating Scales and Assessment in Psychiatry and Mental Health: Springer. 2009;7-35.

51. lannuzzo RW, Jaeger J, Goldberg JF, Kafantaris V, Sublette ME. Development and reliability of the HAM-D/MADRS interview: an integrated depression symptom rating scale. Psychiatry Research. 2006;145(1):21-37.

52. Charous SJ, Kempster G, Manders E, Ristanovic R. The effect of vagal nerve stimulation on voice. The Laryngoscope. 2001;111(11):2028-31.

53. Greenberg BD, Malone DA, Friehs GM, Rezai AR, Kubu CS, Malloy PF, et al. Three-year outcomes in deep brain stimulation for highly resistant obsessivecompulsive disorder. Neuropsychopharmacology. 2006;31(11):2384.

54. Durlak JA. How to select, calculate and interpret effect sizes. Journal of Pediatric Psychology. 2009;34(9):917-28.

55. Christmas DM, Curran S, Matthews K, Eljamel MS. Neurosurgery for mental disorder, vagus nerve stimulation and deep brain stimulation. Psychiatry 2009;8(4):139-43.

56. Osso BD, Dario A, Ciabatti M, Camuri G, Oldani L, Balossi I, et al. Vagus Nerve Stimulation In Treatment-Resistant Bipolar Depression. 2009.

57. Stein DJ, Kupfer DJ, Schatzberg AF. The American Psychiatric Publishing textbook of mood disorders: American Psychiatric Pub. 2007.

58. Miller MD, Reynolds III CF. Depression and Anxiety in Later Life: What Everyone Needs to Know: JHU Press. 2012.

59. Henderson JM. Vagal nerve stimulation versus deep brain stimulation for treatment-resistant depression: show me the data. Clinical Neurosurgery. 2007;54:88.

60. Bewernick BH, Kayser S, Gippert SM, Switala C, Coenen VA, Schlaepfer TE. Deep brain stimulation to the medial forebrain bundle for depression-long-term outcomes and a novel data analysis strategy. Brain Stimulation: Basic, Translational and Clinical Research in Neuromodulation. 2017;10(3):664-71.

61. Naesström M, Blomstedt P, Bodlund O. A systematic review of psychiatric indications for deep brain stimulation, with focus on major depressive and obsessive-compulsive disorder. Nordic Journal of Psychiatry. 2016;70(7):483-91. 